\title{
NOTAS E COMUNICAÇÕES
}

\section{RECURSOS FRUTÍCOLAS NA VÁRZEA E NA TERRA FIRME EM ONZE COMUNIDADES RURAIS DO ALTO SOLIMÕES, AMAZONAS, BRASIL ${ }^{1}$}

\author{
Charles R. CLEMENT ${ }^{2}$, Hiroshi NODA ${ }^{2}$, Sandra do N. NODA ${ }^{3}$, \\ Ayrton L. Urizzi MARTINS ${ }^{4}$, Gleissimar Campelo da SILVA
}

Resumo - A região do Alto Solimões é muito rica em recursos genéticos agrícolas, especialmente frutícolas, mas estes recursos contribuem principalmente para a subsistência em lugar de ter um papel mais amplo no desenvolvimento regional. Um levantamento qualitativo preliminar das espécies frutícolas nativas e exóticas foi executado em 11 comunidades rurais ( 8 caboclas, 3 Ticunas) nos ecossistemas principais (várzea, terra firme). Observou-se 37 fruteiras nativas em 20 familias e 10 fruteiras exóticas em 9 familias; das nativas, 9 foram raras (observadas em 1 ou 2 comunidades) e 3 foram comuns (observadas em 10 ou 11 comunidades); das exóticas, 2 foram raras e as bananas foram encontradas em todas as comunidades, sendo a fruteira mais importante na subsistência e na comercialização. As comunidades com mais acesso imediato à várzea e à terra firme tiveram mais espécies $(22,5 \pm 6,6$ nativas e $6,5 \pm 2,2$ exóticas), que comunidades com acesso apenas à terra firme ( $19,0 \pm 2,2$ nativas e $5,3 \pm 0,5$ exóticas), que por sua vez tiveram mais espécies que comunidades com acesso apenas à várzea $(5 \pm 2$ nativas e $1,5 \pm 0,9$ exóticas).

Palavras-chave - Amazônia, fruteiras, caboclos, Amerindios, barrancos

Fruit Resources of Eleven Rural Communities in the Floodplains and on the Uplands of the Upper Solimões, Amazonas, Brazil

Abstract - The region known as the Upper Solimões is very rich in crop genetic resources, especially of fruits, but these resources contribute principally to subsistence rather than having a more ample importance in regional development. A preliminary qualitative survey of native and exotic fruit species was carried out in 11 rural communities ( 8 traditional peasant, 3 Ticuna Indian) in the principal local ecosystems (floodplain, upland). Thirty-seven native fruits, in 20 families, and 10 exotic fruits, in 9 families were observed; of the natives, 9 were rare (observed in 1 or 2 communities) and 3 were common (observed in 10 or 11 communities); of the exotics, 2 were rare and bananas were found in all communities, being important in both subsistence and trade. Communities with immediate access to both the floodplain and the uplands had more species (22.5 \pm 6.6 natives and $6.5 \pm 2.2$ exotics) than communities with access only to the uplands (19.0 \pm 2.2 natives and $5.3 \pm 0.5$ exotics), which in turn had more species than communities with access only to the floodplain ( $5 \pm 2$ natives and $1.5 \pm 0.9$ exotics).

Key-words - Amazonia, fruits, traditional populations, indigenous populations, bluffs

${ }^{1}$ Apoio financeiro do Projeto de Pesquisa Institucional do INPA no 4.3360 "Coleta, preservação, caracterização e uso de germoplasma tropical" e do Programa Norte de Pós-graduação "Organização social de agricultores familiares no manejo, conservação e utilização de recursos naturais" (FINEP/ CAPES/UA).

${ }^{2}$ Instituto Nacional de Pesquisas da Amazônia - INPA; Cx. Postal 478; 69011-970 Manaus, AM, Brasil

${ }^{3}$ Universidade do Amazonas - UA; Faculdade de Ciências Agrárias; Av. Gal. Rodrigo Otávio Jordão Ramos, 3000; 69077-000 Manaus, AM, Brasil

${ }^{4}$ Instituto Luterano de Ensino Superior de Manaus/ULBRA; Av, Solimões, no 2; Conjunto Atíllio Andreazza - Japiim II; 69077-730 Manaus, AM, Brasil

${ }^{5}$ Movimento de Educação de Base - MEB; Av. Castelo Branco, s/n; 69630-000 Benjamin Constant, AM, Brasil 
A região do Alto Rio Solimões contém sete municípios no extremo oeste do Estado do Amazonas, Brasil, e localiza-se dentro do centro de diversidade genética de cultivos do noroeste amazônico e da região de diversidade genética do Solimões (Clement, 1989, 1999b). É uma das regiões amazônicas com maior número de espécies agrícolas nativas e maior diversidade genética destas espécies, especialmente espécies frutícolas (Clement, 1999a). Este estudo objetivou identificar as espécies fruticolas presentes no Alto Solimões, os ecossistemas em que se encontram e as comunidades rurais que as possuem.

A informação colhida também se relaciona com a relativa facilidade de atender as necessidades básicas das populações humanas nos diferentes ecossistemas regionais, com reflexos sobre sua distribuição atual e passada. Denevan (1996) sugeriu que as populações indigenas ocuparam preferencialmente os barrancos altos ao longo dos principais rios da região, hipótese apoiada pela distribuição de terra preta do índio na Amazônia (Smith, 1995). A preferência para estes locais se devia a sua altura (para visibilidade, defesa, e despreocupação com as enchentes) e acesso imediato a múltiplos ecossistemas (terra firme média e barrancos, várzea baixa, média e alta), com consequências claras para a subsistência (Denevan, 1996). Como corolário do acesso a múltiplos ecossistemas, o número de cultivos manejados deveria ser maior. Este estudo também examina esta hipótese.

Entre 23 de fevereiro e 4 de março de 1996 visitou-se 11 comunidades rurais da região do Alto Rio Solimões, Amazonas, Brasil (Tab. 1). Em cada comunidade, realizou-se um levantamento qualitativo preliminar das espécies frutícolas sensu amplo presentes, com entrevistas não-estruturadas com um ou dois informantes. No sensu amplo inclui-se qualquer espécie cujos frutos são usados, mesmo se não como fruta. O levantamento foi feito durante uma caminhada da beira do rio até uma das roças mais perto das habitações, passando pelo centro da comunidade (exceto no caso de Feijoal, que não tem roças próximas por ser uma comunidade muito velha e grande). $\mathrm{O}$ tempo destas observações e entrevistas variou de duas a três horas; portanto, representam apenas uma amostra da diversidade específica atualmente existente. Não foram feitas observações quantitativas, como recomendado por Alexiades (1996), porque o tempo não permitia. Todas as comunidades também tiveram mandioca e macaxeira (Manihot esculenta), plantas medicinais e diversas hortaliças que não foram levantadas nesta ocasião.

Observou-se 37 espécies frutícolas nativas, de 20 famílias (Tab. 2). Devido à metodologia adotada, este número certamente subestima o número de espécies presentes. Mesmo assim, representa $45 \%$ das 83 espécies frutícolas amazônicas e neotropicais provavelmente presentes na Amazônia em 1500 a.C. (Clement, 1999a). 
Tabela 1. Descrição das comunidades rurais visitadas em 1996 no Alto Solimões, sua localização (com municipio), seus ecossistemas, grupos étnicos e número de famílias.

\begin{tabular}{|c|c|c|c|c|}
\hline Comunidade & Local** & Ecossistema & Grupo Social & Familias \\
\hline 1. Boa Vista & $\begin{array}{l}\text { Paraná de Mauá, llha do Bom } \\
\text { Intento, BC }\end{array}$ & $v-m \& b$ & $\begin{array}{l}\text { caboclo (índio } \\
\text { Cocama?) }\end{array}$ & 10 \\
\hline 2. Novo Paraíso & idem & $v \cdot m \& b$ & Ticuna & 12 \\
\hline 3. $\mathrm{St}^{\mathrm{B}}$ Lucia & $\begin{array}{l}\text { llha do Aramaçá, Rio Solimőes, } \\
\text { Tab. }\end{array}$ & $v-a \& m \& b$ & caboclo & 17 \\
\hline 4. Guanabara II & $\begin{array}{l}\text { Rio Solimões, frente llha do } \\
\text { Aramaçá, BC }\end{array}$ & $\begin{array}{c}\mathrm{tf}-\mathrm{m} \& \mathrm{a} \\
v-\mathrm{m}\end{array}$ & caboclo & 24 \\
\hline 5. Capacete & $\begin{array}{l}\text { Rio Solimões, abaixo llha do } \\
\text { Aramaçá, BC }\end{array}$ & $\underset{v \rightarrow-m}{t f} \&$ a & caboclo & 38 \\
\hline 6. Nova Aliança & $\begin{array}{l}\text { Rio Solimôes, frente llha do } \\
\text { Arariá, BC }\end{array}$ & tf $-a$ & $\begin{array}{l}\text { caboclo (indio } \\
\text { Cocama?) }\end{array}$ & 20 \\
\hline 7. Cidade Nova & $\begin{array}{l}\text { Rio Solimōes, abaixo llha do } \\
\text { Arariá, BC }\end{array}$ & $t f-a$ & Ticuna & 31 \\
\hline 8. Feijoal & $\begin{array}{l}\text { Rio Solimóes, abaixo llha do } \\
\text { Arariá, BC }\end{array}$ & tf $-\underset{v=m}{v} \& a$ & Ticuna & 258 \\
\hline 9. Tupi 2 & $\begin{array}{l}\text { Ressaca do Tupi, Rio } \\
\text { Solimóes, SPO }\end{array}$ & $\begin{array}{l}t f-m \& a \\
v-m \& b\end{array}$ & caboclo & 43 \\
\hline 10. Tupi 1 & idem & $v-m \& b$ & cabocio & 13 \\
\hline 11. Com. Nova & Igarapé Crajari, BC & $\mathrm{tf}-\mathrm{m} \& \mathrm{a}$ & cabocio & 7 \\
\hline
\end{tabular}

* Ecossistemas: várzea (v) - alta (a) e/ou média (m) elou baixa (b); terra firma (tf) - alta (a - equivalente a barranco) e/ou média (m).

** BC - Benjamin Constant, Tab - Tabatinga, SPO - São Paulo de Olivença

Observou-se ainda 10 espécies frutícolas exóticas, de 9 famílias, que não estavam presentes antes de 1500 a.C.

Das 37 nativas, 9 podem ser consideradas raras, pois ocorreram em apenas uma ou duas comunidades; das 10 exóticas, 2 foram raras. Três espécies nativas, cueira, mamoeiro e cubiueiro, e uma exótica, bananeira (sem distinguir entre pacovão e banana de mesa), foram muito comuns (frequência $>0,8$ ), pois são adaptadas tanto à várzea como à terra firme. Além de serem comuns, estas quatro espécies produzem durante a maior parte do ano, contribuindo de forma significante à subsistência das comunidades. As bananas são os principais produtos fruticolas de comercialização, além de serem importante na subsistência.

Comunidades com acesso imediato à várzea e à terra firme tiveram mais espécies, tanto nativas como exóticas, que comunidades com acesso apenas à terra firme, que por sua vez tiveram mais espécies que comunidades com acesso apenas à várzea. As comunidades com ambos ecossistemas em ou adjacente à comunidade tiveram $22,5 \pm 6,6$ espécies frutícolas nativas e $6,5 \pm 2,2$ espécies exóticas, enquanto que as com apenas 
Tabela 2. Espécies frutícolas sensu amplo observadas em 11 comunidades do Alto Solimões, Amazonas, Brasil, em 1996.

\begin{tabular}{|c|c|c|c|c|c|c|c|c|c|c|c|c|}
\hline \multirow[b]{2}{*}{ Espécie, Familia } & \multicolumn{12}{|c|}{ Comunidade (número conforme na Tabel a 1) } \\
\hline & 1 & 2 & 3 & 4 & 5 & 6 & 7 & 8 & 9 & 10 & 11 & Freq. \\
\hline \multicolumn{13}{|l|}{ Fruteiras nativas } \\
\hline Caju (Anacar dium occidentale, Anacardiaceae) & - & - & - & $x$ & $\mathrm{x}$ & $x$ & $x$ & $\mathrm{x}$ & $x$ & $\cdot$ & $x$ & 0,64 \\
\hline Taperibá (Spondias mombim , Anacardi aceae) & - & - & - & $\mathrm{x}$ & - & - & - & $x$ & - & - & - & 0,18 \\
\hline Graviola (Annona muricata , Anonaceae) & $\cdot$ & $x$ & $\cdot$ & $x$ & $x$ & $x$ & $x$ & $x$ & $x$ & - & $x$ & 0,73 \\
\hline Biribá (Rollinia mucosa, Anonaceae) & - & - & $\cdot$ & $\mathrm{x}$ & - & $x$ & $\cdot$ & - & $\mathrm{x}$ & - & $x$ & 0,36 \\
\hline Cuia (Crescentia cujete, Bignoniaceae) & $\mathbf{x}$ & $x$ & $x$ & $x$ & $x$ & $x$ & $\mathbf{x}$ & $\mathrm{x}$ & $x$ & $x$ & - & 0,91 \\
\hline Urucum (Bixa orellana, Bixaceae) & - & $\cdot$ & $\cdot$ & - & $\mathrm{x}$ & $x$ & $\mathrm{x}$ & - & $x$ & - & - & 0,36 \\
\hline Sapota (Quararibea cordata Bombacaceae) & - & $\cdot$ & - & $x$ & - & $x$ & $x$ & $\mathbf{x}$ & $x$ & - & $\mathrm{x}$ & 0,55 \\
\hline Abacaxi (Ananas comosus, Bromeliaceae) & - & - & - & - & - & - & $x$ & $x$ & $x$ & - & $x$ & 0,36 \\
\hline Mamâo (Carica papaya, Caricaceae) & $x$ & $\mathrm{x}$ & - & - & $x$ & $x$ & $\mathrm{x}$ & $x$ & $x$ & $x$ & $x$ & 0,82 \\
\hline Marirana (Couepiasubcordata, Chrysobalanceae) & $\cdot$ & $\cdot$ & $\cdot$ & $\mathrm{x}$ & - & $\cdot$ & - & $\mathbf{x}$ & $x$ & $\cdot$ & - & 0,27 \\
\hline Parinari (Parinari montana, Chrysobalanceae) & - & - & $\cdot$ & - & - & $x$ & - & - & - & - & - & 0,09 \\
\hline Bacurizinho (Rheedia acuminata, Guttiferae) & - & $\cdot$ & - & - & - & $\cdot$ & - & $x$ & - & - & - & 0,09 \\
\hline Bacuri-coroa (Rheedia sp., Guttifer ae) & , & $\cdot$ & - & $\cdot$ & $x$ & $x$ & $\cdot$ & $\mathbf{x}$ & $x$ & $\cdot$ & - & 0,36 \\
\hline Mari (Poraqueiba sericea, Icacinaceae) & - & + & - & $\mathrm{x}$ & - & $x$ & $x$ & $\mathbf{x}$ & $x$ & $\cdot$ & $\mathrm{x}$ & 0,55 \\
\hline Abacate (Persea americana, Lauraceae) & $\cdot$ & - & $\cdot$ & - & - & $\cdot$ & $\cdot$ & $\mathrm{x}$ & $\mathrm{x}$ & $\cdot$ & $x$ & 0,27 \\
\hline Castanha-do-Pará (Bertholletia excelsa, Lecythidaceae) & - & $\cdot$ & - & - & - & $\cdot$ & - & $\mathbf{x}$ & $\cdot$ & $\cdot$ & - & 0,09 \\
\hline Inga (Inga spp., Leguminosae Mimosoi deae) & - & - & - & $x$ & - & $x$ & $\mathrm{x}$ & $x$ & $\mathrm{x}$ & $\cdot$ & $\mathbf{x}$ & 0,55 \\
\hline Mapati (Pourouma cecropiifolia, Moraceae) & - & - & - & $\mathrm{x}$ & - & $x$ & $x$ & $x$ & $x$ & $\cdot$ & $x$ & 0,55 \\
\hline Guabiraba (Campomanesialineatifolia, Myrtaceae) & - & , & $\cdot$ & - & - & - & $\cdot$ & - & $\mathrm{x}$ & $\cdot$ & $x$ & 0,18 \\
\hline cf Camu-camu (Myrciaria sp., Myrtaceae) & - & - & $\cdot$ & - & - & $\cdot$ & - & $x$ & - & - & - & 0,09 \\
\hline Goiaba (Psidium guajava, Myrtaceae) & - & $x$ & $x$ & $\cdot$ & - & $x$ & $\cdot$ & $x$ & $\mathbf{x}$ & - & $\mathrm{x}$ & 0,55 \\
\hline Tucumă (Astrocaryum aculeata, Palmae) & - & - & - & $\mathrm{x}$ & - & - & $\cdot$ & $\mathrm{x}$ & $x$ & $\cdot$ & - & 0,27 \\
\hline Muru-muru (Astrocaryum murumuru, Palmae) & - & - & - & $\mathrm{x}$ & - & - & $\cdot$ & - & $\cdot$ & - & - & 0,09 \\
\hline Pupunha (Bactris gasipaes, Palmae) & $\cdot$ & $\cdot$ & - & $x$ & $x$ & $\mathrm{x}$ & $x$ & $x$ & $x$ & - & $x$ & 0,64 \\
\hline Açai (Euterpe spp., Palmae) & - & $\cdot$ & $\cdot$ & $x$ & $x$ & $x$ & $x$ & $x$ & $x$ & $x$ & $x$ & 0,73 \\
\hline Buriti (Mauritia flexuosa, Palmae) & - & - & $\cdot$ & - & - & $x$ & - & $x$ & $x$ & $\cdot$ & $x$ & 0,36 \\
\hline
\end{tabular}




\begin{tabular}{|c|c|c|c|c|c|c|c|c|c|c|c|c|}
\hline \multirow[b]{2}{*}{ Espécie, Familia } & \multicolumn{12}{|c|}{ Comunidade (número conforme na Tabela 1) } \\
\hline & 1 & 2 & 3 & 4 & 5 & 6 & 7 & 8 & 9 & 10 & 11 & Freq \\
\hline \multicolumn{13}{|l|}{ Fruteiras n ativas (cont.) } \\
\hline Bacaba (Oenocarpus bacaba, Palmae) & $\cdot$ & - & $\cdot$ & $\cdot$ & - & $x$ & $\cdot$ & - & - & - & - & 0,09 \\
\hline Bacabinha (Oenocarpus mapora, Palmae) & $\cdot$ & - & $\cdot$ & $x$ & $x$ & $x$ & $\cdot$ & $x$ & $x$ & $x$ & $x$ & 0,64 \\
\hline Purui (cf. Alibertia edulis, Rubiac eae) & $\cdot$ & $x$ & $\cdot$ & $x$ & $\cdot$ & - & $\rightarrow$ & $x$ & $\cdot$ & - & $=$ & 0,27 \\
\hline Jenipapo (Genipa americana, Rubiaceae) & $\cdot$ & - & $\cdot$ & - & $x$ & $x$ & $x$ & $x$ & $x$ & $x$ & $\cdot$ & 0,55 \\
\hline Maçaranduba (Manilkarahuberi, Sapotaceae) & $\cdot$ & $\cdot$ & , & $\cdot$ & $\cdot$ & $\cdot$ & $\cdot$ & - & $x$ & $\cdot$ & - & 0,09 \\
\hline Abiu (Pouteria caimito, Sapotaceae) & $\cdot$ & $\cdot$ & $\cdot$ & $x$ & - & $x$ & $x$ & $x$ & $x$ & $\cdot$ & $x$ & 0,55 \\
\hline Cubiu (Solanum sessiliflorum, Solanaceae) & $x$ & $x$ & $x$ & $\mathbf{x}$ & $x$ & $x$ & $\cdot$ & $x$ & $x$ & $x$ & $\cdot$ & 0,82 \\
\hline Macambo (Theobromabicolor, Sterculiaceae) & $\cdot$ & - & $\cdot$ & - & $x$ & $\cdot$ & $x$ & $x$ & $\cdot$ & - & $\cdot$ & 0,27 \\
\hline Cacao (Theobroma cacao, Sterculiaceae) & $\cdot$ & - & $\cdot$ & $x$ & $x$ & - & - & $x$ & $\cdot$ & $x$ & $x$ & 0,45 \\
\hline Cupuaçu (Theobromagrandiflorum, Sterculiaceae) & $\cdot$ & $x$ & · & $x$ & $\cdot$ & $\mathbf{x}$ & $x$ & $x$ & $x$ & - & $x$ & 0,64 \\
\hline Cupuí (Theobroma subincanum, Sterculiaceae) & $\cdot$ & - & - & - & $\cdot$ & $\cdot$ & $\mathrm{x}$ & $x$ & $x$ & $\cdot$ & $\cdot$ & 0,27 \\
\hline Total es pécies nativas & 3 & 7 & 3 & 20 & 13 & 22 & 17 & 30 & 27 & 7 & 18 & \\
\hline \multicolumn{13}{|l|}{ Fruteiras exóticas } \\
\hline Manga (Mangifera indica, Anacardiaceae) & $\cdot$ & - & $\cdot$ & $x$ & $x$ & $x$ & - & $x$ & $\cdot$ & $\cdot$ & $x$ & 0,45 \\
\hline Cajarana (Spondias cythera , Anacardi aceae) & $\cdot$ & - & . & - & - & $\cdot$ & - & $x$ & $\cdot$ & $\cdot$ & - & 0,09 \\
\hline Fruta-påo (Artocarpus altilis, Moraceae) & - & - & $\cdot$ & $x$ & $x$ & $\cdot$ & $x$ & $\cdot$ & $x$ & $x$ & $\cdot$ & 0,45 \\
\hline Banana (Musa spp., Musaceae) & $x$ & $x$ & $x$ & $x$ & $x$ & $x$ & $x$ & $x$ & $\mathbf{x}$ & $x$ & $x$ & 1,00 \\
\hline Jambo (Syzygium malaccense, Myrtaceae) & - & $\cdot$ & - & $x$ & $\cdot$ & $x$ & $\mathrm{x}$ & $x$ & $x$ & $x$ & $x$ & 0,64 \\
\hline Carambola (Averrhoa carambola, Oxalidaceae) & - & - & . & - & - & - & $\cdot$ & $x$ & $x$ & - & $x$ & 0,27 \\
\hline Cóco (Cocos nucifera, Palmae) & - & - & - & $x$ & $\cdot$ & $x$ & $x$ & $x$ & $x$ & - & $x$ & 0,55 \\
\hline Pimenta-rei no (Piper nigr um, Piperaceae) & $\cdot$ & - & $\cdot$ & - & $\cdot$ & $\cdot$ & $\cdot$ & $\mathrm{x}$ & $\cdot$ & - & - & 0.09 \\
\hline Café (Coffea sp., Rubiaceae) & $\cdot$ & - & $\cdot$ & $x$ & - & $\cdot$ & - & $x$ & $x$ & - & - & 0,27 \\
\hline Cítricos (Citrus spp., Rutaceae) & - & - & $\cdot$ & $x$ & - & $x$ & $x$ & $x$ & $x$ & $\cdot$ & $x$ & 0,55 \\
\hline Total es péci es exóticas & 1 & 1 & 1 & 7 & 3 & 5 & 5 & 9 & 7 & 3 & 6 & \\
\hline Total es péci es observadas & 4 & 8 & 4 & 27 & 16 & 27 & 22 & 39 & 34 & 10 & 24 & \\
\hline
\end{tabular}


terra firme tiveram $19,0 \pm 2,2$ e 5,3 $\pm 0,5$, respectivamente, e as com apenas várzea tiveram $5 \pm 2$ e $1,5 \pm 0,9$, respectivamente. $\mathrm{O}$ pequeno número de espécies frutícolas na várzea é devido à falta de adaptação da maioria destas espécies a periodos de enchente, especialmente nas várzeas baixa e média tão comum nestas comunidades.

A priori, esperou-se que o grupo étnico seria importante determinante do número de espécies frutícolas observado, porque os Ticuna tem uma longa historia na região. No entanto, isto não foi observado (Figura 1). A falta de importância do grupo étnico para explicar o número de fruteiras talvez seja devido ao intercâmbio de germoplasma entre comunidades rurais, sem preconceito étnico.

Este levantamento preliminar mostrou que o Alto Solimões possue um grande número de recursos frutícolas que poderiam ser melhor aproveitados local e regionalmente, oferecendo uma alternativa potencialmente importante para o desenvolvimento regional. Ainda demostrou que as populações tradicionais, tanto caboclas como indígenas, que localizam suas comunidades nos barrancos de terra firme com acesso para outros ecossistemas possuem uma maior variedade de recursos de subsistência a mão, apoiando a hipótese de Denevan (1996).

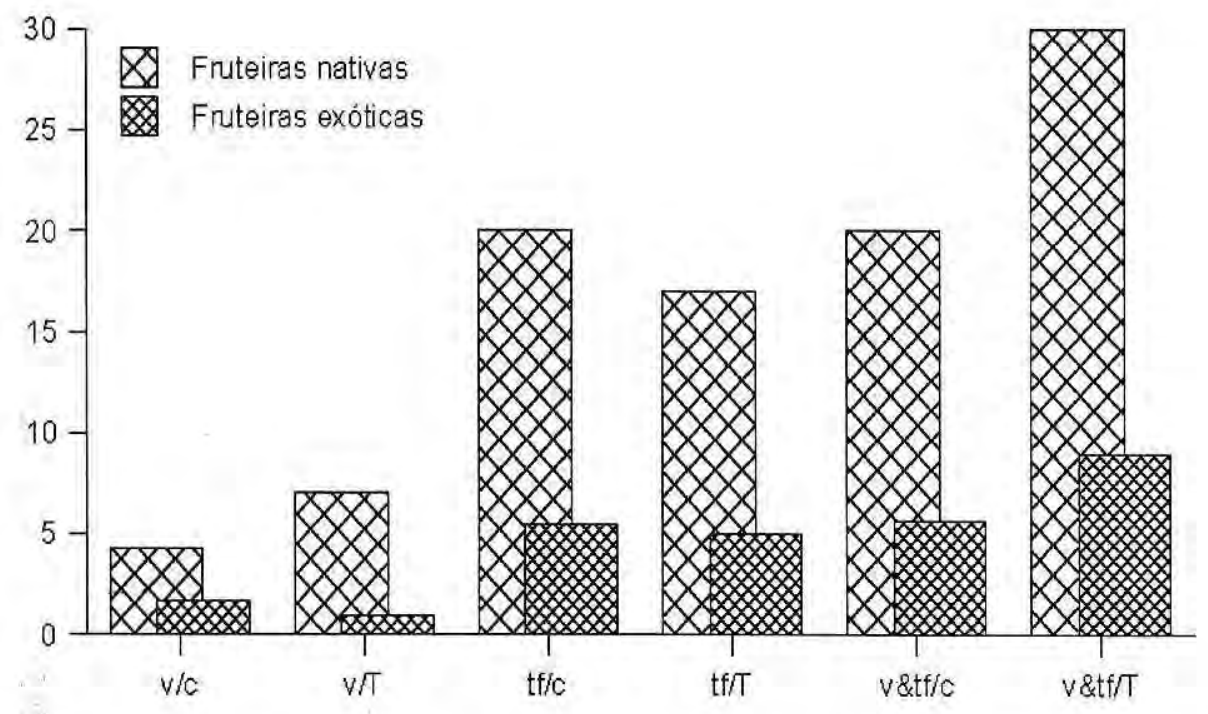

Figura 1. Número médio de espécies frutícolas sensu amplo nativas e exóticas manejadas por 11 comunidades rurais do Alto Solimões, Amazonas, Brasil, em termos de ecossistemas ( $\mathrm{v}=$ várzea; $\mathrm{tf}=$ terra firme) e grupo étnico $(\mathrm{c}=$ caboclo; $\mathrm{T}=$ Ticuna $)$. 


\section{Bibliografia citada}

Alexiades, M.N. (Ed.) 1996. Selected guidelines for ethnobotanical research: a field manual. New York Botanical Garden, New York. 306 p.

Clement, C.R. 1989. A center of crop genetic diversity in western Amazonia. BioScience, 39:624-631.

Clement, C.R. 1997. Environmental impacts of, and biological and socio-economic limitations on new crop development in Brazilian Amazonia. In: Smartt, J.; Haq, N.N. (Eds.). Domestication, Production and Utilization of New Crops: Practical Approaches. International Centre for Underutilised Crops, University of Southampton, Southampton, England, UK. pp. 134-146.

Clement, C.R. 1999a. 1492 and the loss of Amazonian crop genetic resources. I. The relation between domestication and human population decline. Economic Botany, 53(2):188-202.

Clement, C.R. 1999b. 1492 and the loss of Amazonian crop genetic resources. II. Crop biogeography at contact. Economic Botany, 53(2):203-216.

Denevan, W. M. 1996. A bluff model of riverine settlement in prehistoric Amazonia. Annals Association American Geographers, 86(4):654_681.

Smith, N.J.H. 1995. Human-induced landscape changes in Amazonia and implications for development. In: Turner II, B. L.; Gómez Sal, A.; González Bernáldez, F; di Castri, F. (Eds.). Global land use change - A perspective from the Columbian Encounter. Consejo Superior de Investigaciones Científicas, Madrid. pp. 221-251. 